Cahiers d'Études Germaniques

$77 \mid 2019$

Histoire des juifs et des juives d'Allemagne

(1867-1933)

\title{
Le sionisme comme fait de civilisation allemande
}

Zionismus als Bestandteil deutscher Geschichte und Kultur

Zionism and German Culture

Olivier BAISEZ

(2) OpenEdition

Journals

Édition électronique

URL : https://journals.openedition.org/ceg/7568

DOI : $10.4000 /$ ceg.7568

ISSN : 2605-8359

Éditeur

Presses Universitaires de Provence

Édition imprimée

Date de publication : 25 septembre 2019

Pagination : 159-170

ISBN : 979-10-320-0234-6

ISSN : 0751-4239

Référence électronique

Olivier BAISEZ, «Le sionisme comme fait de civilisation allemande », Cahiers d'Études Germaniques [En ligne], 77 | 2019, mis en ligne le 25 mars 2021, consulté le 15 juin 2021. URL : http:// journals.openedition.org/ceg/7568; DOI : https://doi.org/10.4000/ceg.7568

Tous droits réservés 


\title{
Le sionisme comme fait de civilisation allemande
}

\author{
Olivier BAISEZ \\ Université Paris 8 Vincennes - Saint-Denis / « Les mondes allemands » (EA 1577)
}

\section{Introduction}

Cet article s'articule autour de trois exemples qui illustrent, sinon démontrent, le profond ancrage du sionisme dans un contexte allemand, et justifient qu'on en traite comme d'un objet de la « civilisation allemande » à part entière. Par fait de civilisation allemande, j'entends un phénomène social, politique et culturel dont l'inscription dans un environnement social, politique et culturel allemand lui est pour ainsi dire consubstantielle.

Dans le cas du sionisme, qui se présente comme une expression du sentiment national juif et prétend offrir une solution à la " question juive ${ }^{1}$ ", une telle affirmation a de quoi surprendre au premier abord. Or, la manière de concevoir le programme du sionisme, ses objectifs et ses méthodes, a été déterminée par le contexte allemand ${ }^{2}$. Le " sionisme allemand » n'est pas seulement le sionisme germanophone, l'allemand étant jusqu'en 1911 (date du Xe congrès, le premier à débattre majoritairement en hébreu) la lingua franca ou langue véhiculaire du mouvement, ainsi que la langue de formation de ses principaux dirigeants. La notion de sionisme allemand existe au sein du sionisme en général, aussi bien dans les textes contemporains que dans l'historiographie, et désigne la branche allemande du mouvement sioniste international, qui en est jusqu'à la Première Guerre mondiale la branche maîtresse. Institutionnellement parlant, elle s'incarne dans la fédération sioniste allemande (Zionistische Vereinigung für Deutschland, ZVfD). Elle n'est pas la plus importante en nombre, mais la plus influente des fédérations nationales, du moins jusqu'à la Première Guerre mondiale et le déplacement du centre de gravité du mouvement d'abord vers des pays neutres (Danemark, Pays-Bas) puis vers Londres. Le fait le plus éloquent à cet égard est que la ZVfD et l'Organisation sioniste mondiale (OSM) partagent de 1905 à 1914

1. Le titre complet du livre publié par Theodor Herzl en 1896 est Der Judenstaat. Versuch einer modernen Lösung der Judenfrage. Sur la « question juive » parmi les autres grandes questions formulées au XIXe siècle, cf. Holly Case, The Age of Questions, Princeton, Princeton University Press, 2018.

2. Cf. Olivier Baisez, Architectes de Sion. La conception par les sionistes allemands de la colonisation juive en Palestine (1896-1919), Paris, Hermann, 2015. 
la même adresse, à Cologne puis Berlin. En outre, David Wolffsohn (1855-1914) et Otto Warburg (1859-1938), les deux successeurs de Theodor Herzl (1860-1904) à la présidence de l'OSM, sont tous deux issus des rangs de la ZVfD.

II faut bien garder à l'esprit que le sionisme est largement minoritaire parmi les Juifs allemands pendant toute la période considérée, et l'émigration en Palestine encore bien plus, bien qu'elle ait été officiellement déclarée partie intégrante du « programme de vie » de tout sioniste depuis l'adoption de la résolution de Posen (Poznań) par les délégués de la ZVfD en 1913. Rares, cependant, furent ceux qui franchirent le pas avant $1933^{3}$.

Les trois exemples retenus l'ont été pour leur complémentarité. Même si, dans les trois cas, des indications de nature biographique sont indispensables pour bien situer le propos, le plan suivra le principe suivant : un homme, une œuvre, une institution. L'homme, ce sera Otto Warburg. L'œuvre, Der Untergang der deutschen Juden, de Felix Theilhaber (1884-1956). Et l'institution, la revue Der Jude fondée et dirigée par Martin Buber (1878-1965). L'année 1911 pourrait faire office de dénominateur commun biographique, Warburg accédant à cette date à la présidence de I'OSM, Theilhaber publiant Der Untergang der deutschen Juden et s'engageant comme médecin militaire auprès du Croissant rouge ottoman, Buber publiant son ouvrage le plus important avant-guerre, Drei Reden über das Judentum. Enfin, chacun des trois exemples illustre la consubstantialité du sionisme allemand et de son contexte intellectuel dans un domaine particulier, les sciences naturelles et l'expertise coloniale, la médecine et la démographie, la philosophie et le nationalisme, qui sont autant de voies d'approche classiques par lesquelles on aborde l'histoire culturelle allemande.

\section{Otto Warburg, ou : de la noix de muscade à la présidence de l'Organisation sioniste mondiale}

Otto Warburg descend d'une célèbre famille juive ayant fait fortune dans la banque et le commerce ${ }^{4}$. L'historien de l'art Aby Warburg et le prix Nobel de physiologie ou médecine Otto Warburg, son homonyme, sont ses cousins éloignés. II ne reçut aucune éducation spécifiquement juive. Après avoir obtenu son Abitur à Hambourg, il entreprit des études de botanique, zoologie et chimie à l'université de Bonn, puis à Berlin. Ce fut ensuite l'université de Strasbourg qui lui décerna

3. Le volume total de l'émigration judéo-allemande en Palestine avant 1933 est généralement estimé à deux mille personnes au maximum. II n'existe à ce jour aucune monographie sur le sujet. Sur l'émigration judéo-allemande en Palestine, cf. Mordechai Eliav, « German Jews' Share in the Building of the National Home in Palestine and the State of Israel », The Leo Baeck Institute Year Book 30 (1), 1985, p. 255-263.

4. Sur la famille Warburg, cf. Ron Chernow, The Warburgs: The Twentieth-Century Odyssey of a Remarkable Jewish Family, New York, Random House, 1993. Sur la vie d'Otto Warburg, le site internet du Otto Warburg Minerva Center for Agricultural Biotechnology à I'Université hébraïque de Jérusalem fournit une longue notice biographique : [http://departments.agri.huji.ac.il/biotech/ otto.htm], dernière consultation le 4 mai 2019. 
le doctorat en 1883. Tout en publiant ses premiers textes scientifiques, Warburg prépara soigneusement une expédition botanique de longue durée : de 1885 à 1889, il parcourut une grande partie de l'Asie méridionale et de l'Océanie. De retour à Berlin, il travailla à la publication de ses recherches et à son habilitation. II devint en 1892 membre de la prestigieuse académie Leopoldina (Halle).

Son expérience de l'Asie tropicale et équatoriale le fit entrer en contact avec les milieux coloniaux allemands (le début de sa carrière professionnelle coïncide avec l'entrée de l'Allemagne dans la course aux colonies en 1884), où son expertise botanique et surtout agronomique était recherchée. En 1897, il publia un traité sur la noix de muscade ${ }^{5}$ et fonda la revue d'agronomie tropicale Der Tropenpflanzer. Zeitschrift für tropische Landwirtschaft, qu'il dirigea pendant un quart de siècle avec le professeur Ferdinand Wohltmann. La revue était l'organe du Kolonial-wirtschaftliches Komitee, dont il était également membre fondateur ${ }^{6}$, et qui constituait la commission économique de la Deutsche Kolonialgesellschaft $\left(D_{K G}{ }^{7}\right)$, la principale organisation du lobby colonial allemand. II s'impliqua par ailleurs dans diverses sociétés commerciales exploitant des plantes industrielles ou défendant les intérêts allemands à l'étranger, notamment la DeutschLevantinische Baumwoll-Gesellschaft ou la Deutsch-Asiatische Gesellschaft. Un article est consacré à Warburg dans le Deutsches Kolonial-Lexikon ${ }^{8}$.

Parallèlement à ces activités, Warburg s'engagea dans le mouvement sioniste et s'intéressa en particulier aux aspects pratiques de la colonisation juive en Palestine. Il rejoignit le mouvement par l'intermédiaire de son beau-père, Gustav Gabriel Cohen (1830-1906 ${ }^{\circ}$, banquier à Hambourg et sioniste de la toute première heure (il avait écrit Die Judenfrage und die Zukunft dès la fin des années 1880 et défendait l'idée d'une colonisation juive sur l'île de Chypre ${ }^{10}$ ), qui lui fit rencontrer Theodor Herzl, avec qui Cohen s'était lié d'amitié, en $1897^{11}$.

Herzl vit en Warburg un expert bien introduit dans les milieux scientifiques et coloniaux allemands, dont les connaissances pourraient devenir utiles à la réalisation du programme sioniste tel qu'il avait été adopté par le ler congrès à Bâle en 1897. Par ailleurs, Warburg était depuis 1894 membre de l'association

5. Otto Warburg, Die Muskatnuss, ihre Geschichte, Botanik, Kultur, Handel und Verwerthung, Leipzig, Engelmann, 1897.

6. La personnalité centrale de ce comité était l'industriel Karl Supf (1855-1915).

7. Le KWK revendique une compétence scientifique et technique, indépendante des débats de politique coloniale. C'est à partir de 1902 qu'il fait officiellement fonction, dans un rôle consultatif, de commission économique de la DKG.

8. Heinrich Schnee (éd.), Deutsches Kolonial-Lexikon, Leipzig, Quelle \& Meyer, 1920, 3 vol., ici vol. III, p. 675 .

9. Daniel Hoffmann, « Cohen, Gustav Gabriel », Institut für die Geschichte der deutschen Juden (éd.), Das jüdische Hamburg, en ligne : [http://www.dasjuedischehamburg.de/inhalt/cohen-gustavgabriel], dernière consultation le 10 février 2019.

10. Gustav Gabriel Cohen, Die Judenfrage und die Zukunft, Hamburg, Philipsen, 1896. (Impressions préalables à compte d'auteur.)

11. Theodor Herzl, Tagebücher 1895-1904, Berlin, Jüdischer Verlag, 1923, vol. 2, p. 27. Herzl évoque d'agréables conversations nocturnes autour d'une bouteille de vin français pendant le ler congrès. 
berlinoise Esra, qui promouvait l'installation de Juifs en Palestine ${ }^{12}$ et s'inscrivait dans la mouvance « pratique » du sionisme, celle qui, en matière de colonisation, défendait une politique des petits pas et du fait accompli, sans attendre d'hypothétiques garanties juridiques.

Il est à noter que l'épouse d'Otto Warburg, Anna (1882-1967), a elle-même présidé le Verband jüdischer Frauen für Kulturarbeit in Palästina, couramment appelé Kulturverband, fondé en 1907 à Berlin (et officiellement déclaré au VIII congrès la même année à La Haye). La quasi-totalité des fondatrices étaient les épouses de personnalités du sionisme allemand. L'association soignait cependant son ouverture vers des milieux non sionistes mais susceptibles de s'intéresser au sort des femmes juives en Palestine, et faisait donc preuve, du point de vue social, de ce qu'on pourrait appeler une grande ouverture horizontale. L'ouverture verticale, en revanche, était presque nulle : il s'agit d'un exemple typique de bienfaisance bourgeoise, assez similaire aux « bonnes œuvres » chez les catholiques français. Le Kulturverband affiche sa volonté de «civiliser » ou en tout cas d'éduquer les femmes du Yishouv ${ }^{13}$, surtout les "Orientales » (aux deux sens du terme, purement géographique ou culturel), pour en faire de bonnes épouses et de bonnes servantes, voire des infirmières. L'association connut un rapide succès international grâce à l'entregent de ses dirigeantes, et créa de multiples antennes locales à l'étranger ${ }^{14}$.

En 1899-1900, Warburg entreprit son premier voyage en Orient (soit quelques mois après les fameux voyages en Palestine de l'empereur Guillaume II et de Theodor Herzl). II visita l'Anatolie et le Levant, se renseigna sur les possibilités de développement agricole et rencontra les personnalités sionistes présentes sur place, dont Selig Eugen Soskin (1873-1959 $\left.{ }^{15}\right)$. Par la suite, Warburg fit partie des commissions d'expertise chargées d'évaluer la faisabilité de la colonisation juive dans les deux territoires proposés successivement par les autorités coloniales britanniques en réponse aux sollicitations de Herzl : la côte nord du Sinaï (début 1903) et l' "Ouganda » (fin $\left.1904^{16}\right)$. À partir du congrès sioniste de 1903, Warburg dirigea les activités pratiques au sein de l'Organisation sioniste, en qualité de président de la Commission pour l'exploration de la Palestine en vue de son

12. Le nom complet de l'association était Esra - Verein zur Unterstützung ackerbautreibender Juden in Palästina und Syrien. Cf. Jehuda Reinharz (éd.), Dokumente zur Geschichte des deutschen Zionismus 1882-1933, Tübingen, Mohr Siebeck, 1981, p. 7-9.

13. Le terme Yishouv (hébr. ישוב) sert à désigner la population juive de Palestine avant la fondation de l'État d'Israël en 1948.

14. Barbara Schäfer, Berliner Zionistenkreise. Eine vereinsgeschichtliche Studie, Berlin, Metropol Verlag, 2003, p. 85-88.

15. Derek J. Penslar, «Zionism, Colonialism and Technocracy. Otto Warburg and the Commission for the Exploration of Palestine, 1903-7 », Journal of Contemporary History 25 (1), 1990, p. 143-160; Id., «Technical Expertise and the Construction of the Rural Yishuv, 1882-1948 », Jewish History 14 (2), 2000, p. 201-224.

16. Olivier Baisez, "Greater Palestine" as a German-Zionist Idea before the British Mandate Period ", The Leo Baeck Institute Year Book 61 (1), 2016, p. 7-24. Derek J. Penslar, Zionism and Technocracy. The Engineering of Jewish Settlement in Palestine, 1870-1918, Bloomington, Indiana University Press, 1991, p. 64. 
développement économique (Kommission zur Erforschung Palästinas, souvent abrégé en Palästina-Kommission ${ }^{17}$ ). II y travailla notamment avec Soskin et Franz Oppenheimer (1864-1943); les trois hommes coéditèrent la revue Altneuland. Monatsschrift für die wirtschaftliche Erschließung Palästinas pendant trois ans (1904-1906).

Après la disparition subite de Herzl en juillet 1904, Warburg fit partie avec Max Nordau (1849-1923) et David Wolffsohn du triumvirat chargé d'assurer l'intérim à la présidence de l'Organisation sioniste. Le courant « pratique » dirigé par Warburg devait alors lutter contre les réticences du courant «politique ». Wolffsohn, le chef de file de cette seconde tendance, succéda finalement à Herzl, Warburg devant se contenter d'une vice-présidence ${ }^{18}$. Dans sa volonté d'entreprendre des démarches pratiques pour favoriser la colonisation juive en Palestine, Warburg se heurta constamment au manque de moyens financiers et chercha à éveiller l'intérêt d'investisseurs. Son projet d'installer des colons juifs sur des exploitations cotonnières le long du Bagdadbahn en 1904 s'inscrivait dans cette approche, mais ne convainquit pas les autres dirigeants sionistes. Quoi qu'il en soit, avec un tel projet, Warburg faisait converger ses intérêts scientifiques avec ses multiples engagements institutionnels et argumentait à la fois en partisan de l'impérialisme allemand et de la colonisation sioniste (bien que l'implantation en dehors de la Palestine biblique ait toujours été considérée avec une grande méfiance par une majorité de délégués sionistes ${ }^{19}$ ), mais aussi, plus généralement, de la « productivisation » ou de la "normalisation » des Juifs, c'est-à-dire de leurs activités professionnelles souvent jugées trop éloignées du travail de la terre ${ }^{20}$.

Pour la plupart des projets auxquels il participe, Warburg est contraint de puiser dans ses ressources personnelles. Dans les années 1900, il est à l'initiative de nombreuses décisions et joue un rôle crucial dans l'organisation du sionisme, y compris en Palestine. C'est lui, par exemple, qui recommande Arthur Ruppin (18761943) et Jakob Thon (1880-1950) pour prendre en charge le Bureau palestinien que l'Organisation sioniste ouvre à Jaffa afin de superviser les acquisitions foncières, et dont la création est décidée en 1907. Deux ans auparavant, sa Commission pour la Palestine avait été une force motrice dans la fondation de l'école d'arts appliqués «Bezalel » à Jérusalem. Warburg est aussi à l'origine de la Palestine Land-Development Company, créée en 1908, et dont les opérations sont contrôlées sur place par Ruppin. Enfin, le Fonds des oliviers (Ölbaumspende) et la forêt Herzl (Herzlwald), conçus comme des outils de substitution à l'impôt et fonctionnant sur la base de collectes de dons, pour tisser des liens forts entre les

17. Penslar, «Zionism, Colonialism and Technocracy ».

18. Walter Laqueur, Histoire du sionisme, Paris, Calmann-Lévy, 1973, p. 160-231.

19. Otto Warburg, « Die jüdische Kolonisation in Nord-Syrien auf Grundlage der Baumwollkultur im Gebiete der Bagdad-Bahn », Altneuland 1 (7), 1904, p. 193-199 (partie 1); 1 (8), p. 232-240 (partie 2); 1 (9), p. 268-278 (partie 3). Voir Baisez, Architectes de Sion, p. 364-365.

20. On fait généralement remonter l'idée de « productiviser » les Juifs au texte de Christian Wilhelm Dohm: Warburg comme Oppenheimer siégaient au bureau de la Verein zur Beförderung der Bodenkultur unter den Juden. L'idée de productiviser les Juifs est généralement reliée au texte de Christian Wilhelm Dohm, Über die bürgerliche Verbesserung der Juden (1781). 
Juifs d'Allemagne et la colonisation juive en Palestine, ont été lancés largement à son initiative à partir de $1904^{21}$.

Après avoir obtenu l'assentiment de la plupart des partisans du sionisme " pratique ", notamment les leaders sionistes d'Europe orientale et de Russie ${ }^{22}$, Warburg fut élu à la présidence de l'Organisation sioniste mondiale par le Xe congrès en 1911. Le siège de l'organisation fut alors transféré de Cologne à Berlin. Warburg augmenta les marges de manœuvre de Ruppin en Palestine. Les partisans de la colonisation immédiate durent cependant encore lutter contre les réticences au sein des établissements bancaires du mouvement sioniste (Jewish Colonial Trust, Anglo-Palestine Company). À partir de 1914, la guerre compliqua grandement les opérations en Palestine, entraîna la délocalisation des principales institutions sionistes vers des pays neutres, et avec la perte des colonies allemandes, Warburg se trouva privé non seulement de son champ de recherche privilégié, mais perdit aussi l'argent investi dans des entreprises coloniales. La publication de la revue Der Tropenpflanzer dut cesser après 1921, et l'hyperinflation de 1923 entama encore davantage sa fortune personnelle. Pendant et après la guerre, Warburg utilisa ses relations au plus haut niveau dans la classe politique allemande pour intervenir en faveur des Juifs de Palestine. II partagea ensuite son temps entre l'Allemagne et la Palestine, où il contribua à fonder la station agricole expérimentale de Rehovot en 1919 (il la dirigea à partir de 1921). En 1925, quand I'Université hébraïque de Jérusalem ouvrit ses portes, Warburg y fut élu à la chaire de botanique et à la direction de l'institut de sciences naturelles et fut à l'origine du jardin botanique national sur le campus du mont Scopus ${ }^{23}$. II maintint cependant sa résidence principale en Allemagne, en raison de la santé fragile de son épouse. Toutefois, il se rendait régulièrement en Palestine, même après son départ en retraite en 1933. Ses enfants émigrèrent en Palestine après l'arrivée au pouvoir des nazis, lui mourut à Berlin en $1938^{24}$.

L'itinéraire de Warburg illustre au plus haut degré la tentative de concilier les exigences du patriotisme allemand et du sionisme par l'intermédiaire de l'expertise technocratique et de projets de colonisation visant explicitement à faire d'une pierre deux coups. II montre aussi à quel point l'issue de la Première Guerre mondiale a représenté une rupture : solide allié de l'Empire ottoman et pays possédant une importante population juive, l'Allemagne était apparue jusque-là comme la puissance dont les sionistes devraient rechercher le soutien. Après l'invasion du Levant par l'armée britannique et la déclaration Balfour du

21. Penslar, Zionism and Technocracy, p. 73-79. Michael Berkowitz, Zionist Culture and West European Jewry before the First World War, Chapel Hill, University of North Carolina Press, 1996, p. 184-185.

22. Notamment à l'occasion du IX'e congrès tenu à Hambourg en décembre 1909, cf. Reinharz, Dokumente, p. 85.

23. On a pu parler de « sionisme botanique » à propos de Warburg, cf. Frank Leimkugel, Botanischer Zionismus: Otto Warburg (1859-1938) und die Anfänge institutionalisierter Naturwissenschaften in „Erez Israel", Berlin, Botanischer Garten und Botanisches Museum, 2005. Voir aussi : Dana von Suffrin, Pflanzen für Palästina. Otto Warburg und die Naturwissenschaften im Jischuw. Tübingen, Mohr Siebeck, 2019.

24. Sur Warburg voir notamment Etan Bloom, Arthur Ruppin and the Production of Pre-Israeli Culture, Boston/Leyde, Brill, 2011, p. 133-141. 
2 novembre 1917, cet édifice de conjectures et de projets s'effondra. Londres avait en quelque sorte remplacé Berlin.

\section{Der Untergang der deutschen Juden de Felix Theilhaber, ou : I'assimilation comme évaporation}

Né en 1884 à Bamberg, en Franconie, fils d'Adolph Theilhaber, un gynécologue obstétricien qui faisait autorité dans sa discipline et portait le titre de Bayrischer Hofrat, Felix Aron Theilhaber étudia à son tour la médecine aux universités de Berlin et de Munich. Il se spécialisa en dermatologie et obtint le doctorat en 1910, avec une thèse intitulée Der Zusammenhang der sozialen Stellung und der Rasse mit der Entstehung der Uteruscarcinome, ou s'annonçait déjà son intérêt pour les questions d'hygiène sociale et « raciale » (Rassenhygiene), dans le langage de l'époque, ainsi qu'à la sexologie, domaine dans lequel il fit œuvre de pionnier en prônant la « réforme sexuelle » (contrôle des naissances dans les familles pauvres, refus de la criminalisation de l'avortement et de l'homosexualité).

Theilhaber commença ses activités de militant sioniste dès l'université. Il dirigea à Munich la section locale des étudiants sionistes et fonda l'association sportive Makkabi locale. II dirigea aussi pendant trois ans (1908-1910) la publication de la revue Palästina. Zeitschrift für den Aufbau Palästinas. Il est cependant surtout connu pour son étude démographique, qui est aussi le fruit de la fréquentation des cours de Georg von Mayr (1841-1925) à Munich, sur "le déclin des Juifs allemands »(Der Untergang der deutschen Juden. Eine volkswirtschaftliche Studie) parue en 1911, dans laquelle il décrivait comment la baisse du taux de natalité, les « mariages mixtes » ou l' « exogamie » (Mischehen) et la migration vers les villes risquaient de conduire à la dissolution des Juifs allemands dans la société allemande. À ses yeux, le sionisme et la migration vers la Palestine représentaient la seule issue. Le ton alarmiste du livre, qu'on qualifierait aujourd'hui volontiers de décliniste, joua un rôle non négligeable dans son succès ${ }^{25}$.

Ce livre, paru la même année que celui de Ruppin intitulé Die Juden der Gegenwart ${ }^{26}$, un grand tableau statistique de la population juive mondiale, est représentatif d'un intérêt profond de savants juifs pour les problèmes de la race, conçue comme un corps collectif ${ }^{27}$. Theilhaber fait en effet partie de ces personnalités sionistes qui, comme Oppenheimer ou Ruppin, ont participé

25. L'ouvrage fut réédité en 1921. Sur la rhétorique « décliniste » dans le sionisme allemand, cf. Stefan Vogt, "Between Decay and Doom: Zionist Discourses of Untergang in Germany, 1890 to 1933 », in Steven E. Aschheim, Vivian Liska (dir.), The German-Jewish Experience Revisited, Berlin, De Gruyter, 2015, p. 75-102.

26. Arthur Ruppin, Die Juden der Gegenwart. Eine sozialwissenschaftliche Studie, Köln, Jüdischer Verlag, 1911.

27. Cf. John M. Efron, Defenders of the Race: Jewish Doctors and Race Science in Fin-de-Siècle Europe, New Haven, Yale University Press, 1994 ; Mitchell B. Hart, Social Science and the Politics of Modern Jewish Identity, Stanford, Stanford University Press, 2000; Ulrich Charpa, Ute Deichmann (dir.), Jews and Sciences in German contexts, Tübingen, Mohr Siebeck, 2007. 
au débat sur la " race juive » pendant les premières décennies du XXe siècle ${ }^{28}$. Convaincu de l'existence de différences essentielles qui caractériseraient les Juifs en tant que race, il en fait une interprétation positive, en faveur des Juifs ${ }^{29}$. II prend position en faveur de mesures $d^{\prime}$ " "hygiène raciale » telles que le soutien financier aux familles nombreuses. Il est à noter que son propos mobilise des arguments à la fois biologiques, ou médicaux, et sociaux et s'inscrit dans un courant eugéniste influencé par le darwinisme social, où figurent aussi d'autres personnalités sionistes comme Ruppin, Elias Auerbach (1882-1971), Aron Sandler (1879-1954) ou Ignaz Zollschan (1877-1948). À l'instar de Ruppin, Theilhaber considérait les Juifs à la fois comme une des plus anciennes et comme la plus moderne des nations, c'est-à-dire la plus marquée par la vie citadine et le capitalisme, et par les pathologies qui en résulteraient. Cette approche " environnementaliste » entendait aussi réfuter le déterminisme biologique des antisémites, ce qu'on trouve aussi chez Oppenheimer, médecin lui aussi ${ }^{30}$.

Der Untergang der deutschen Juden présente un schéma temporel observable dans maints autres textes de sionistes allemands : d'abord la valorisation du passé juif; ensuite l'affirmation que ce qui en faisait la valeur est absent dans le présent; enfin l'assurance que la colonisation sioniste le restaurera dans l'avenir. En termes médicaux, c'est une sorte de triptyque ou de courbe en $U$ : partant d'un état de santé et vitalité, on tombe dans la maladie et l'apathie, mais il existe un remède pour retrouver santé et vitalité. S'inscrivant dans une tradition déjà ancienne au début du XXe siècle, le livre de Theilhaber constitue en fait un plaidoyer pour la régénération ${ }^{31}$ et la normalisation de l'existence des Juifs, c'est-à-dire, du point de vue du médecin, l'assainissement voire la guérison. Pour cela, il faudrait un sentiment national vigoureux, un territoire, une langue, des traditions, des intérêts politiques et économiques communs ${ }^{32}$.

L'année même de la parution de son ouvrage, Theilhaber s'engagea comme médecin militaire auprès du Croissant Rouge turc pendant la guerre italo-turque de 1911-1912, ainsi que les guerres balkaniques qui ont immédiatement suivi. À cette occasion, il voyagea pour la deuxième fois jusqu'en Palestine, après un premier séjour en 1906. Il dut ensuite rentrer en Allemagne. Pendant la Première Guerre mondiale, il servit comme officier de santé dans l'armée impériale allemande. Après la guerre, au cours de laquelle les combattants juifs durent faire face au soupçon de déloyauté, il voulut contribuer à leur réhabilitation, à la manière du Reichsbund jüdischer Frontsoldaten, en choisissant de consacrer un

28. Veronika Lipphardt, Biologie der Juden. Jüdische Wissenschaftler über "Rasse“ und Vererbung 1900-1935, Göttingen, Vandenhoeck \& Ruprecht, 2008.

29. Olivier Baisez, «La race, la souche et le peuple : Franz Oppenheimer, le sionisme et l'identité des Juifs allemands », in Dorothea Bohnekamp, Penser les identités juives dans l'espace germanique, XIXe-XXe siècles, Rennes, Presses universitaires de Rennes, 2015, p. 61-77. Sur la distinction entre racialisme et racisme, cf. Pierre-André Taguieff, «Éric Voegelin, 1933 : un philosophe face à l'idée de race et au racisme », in Éric Voegelin, Race et État, Paris, Vrin, 2007 [1933], p. 7-88.

30. Baisez, «La race, la souche et le peuple».

31. Voir notamment : Max Nordau, Entartung, Berlin, Duncker, 1892. Nordau était médecin de formation lui aussi.

32. Hart, Social Science, p. 85, p. 87. 
livre aux pilotes juifs dans l'aviation allemande, inscrivant ainsi les Juifs dans la geste de ces « chevaliers des temps modernes » que furent les premiers pilotes de combat ${ }^{33}$.

Par ailleurs, Theilhaber est connu pour avoir fondé en 1913 la Société pour la réforme sexuelle (Gesellschaft für Sexualreform, abrégé en GeSex), et en 1928, il lança la publication périodique Zeitschrift für Sexualhygiene. En 1930, il contribua à fonder la première clinique berlinoise consacrée à la planification familiale et à l'éducation sexuelle. Theilhaber quitta l'Allemagne en 1935, après y avoir perdu le droit d'exercer la médecine, et ouvrit un cabinet en Palestine. Avec des confrères, il y fonda une caisse privée d'assurance-santé, un projet qu'il nourrissait depuis 1910 au moins ${ }^{34}$. Il s'éteignit en 1956 à Tel Aviv.

\section{La revue Der Jude, ou : le grand écart permanent entre l'universel et le particulier}

Voix du sionisme dit «culturel », davantage soucieux de la « renaissance » spirituelle et créative du judaïsme ${ }^{35}$ que des modalités pratiques de la colonisation, le mensuel Der Jude, lancé en 1916 et publié jusqu'en 1928, fut une institution majeure du sionisme en général, et du sionisme allemand en particulier. Sous l'égide de Martin Buber $^{36}$, Der Jude (dont le tirage de trois à cinq mille exemplaires au plus fort de sa diffusion est important pour une publication de ce type) entendait surmonter les divisions du monde juif par l'affirmation de son unité et de sa vitalité culturelle intrinsèque, dont les populations juives d'Europe orientale, « révélées » aux Juifs d'Allemagne par la guerre sur le front russe, faisaient encore sentir la pulsation. En effet, l'expérience de guerre et des modes de vie traditionnels remirent en question la conscience de soi de nombreux Juifs en Europe centrale, notamment en Allemagne ${ }^{37}$. Les problèmes politiques, économiques et sociaux étaient cependant loin d'être absents des pages de la revue, et plusieurs contributeurs de périodiques plus spécialisés dans ces domaines ont présenté leurs arguments au lectorat de ce mensuel ${ }^{38}$.

33. Jüdische Flieger im Weltkrieg: ein Buch der Erinnerung, Berlin, Lamm, 1919.

34. Cf. Felix Theilhaber, "Ein Beitrag zur Lösung der Geldfrage im Zionismus », Die Welt 21, 1910, p. 512-515. Dès cette époque, Theilhaber fait remarquer qu'il s'intéresse à la question d'une assurance-vie juive et sioniste depuis plusieurs années déjà.

35. Delphine Bechtel, La Renaissance culturelle juive en Europe centrale et orientale, 1897-1930, Paris, Belin, 2002.

36. Dominique Bourel, Martin Buber, Sentinelle de l'humanité, Paris, Albin Michel, 2015.

37. Voir notamment l'ouvrage d'Arnold Zweig, Das ostjüdische Antlitz, Berlin, Welt-Verlag, 1920. Cf. Sander L. Gilman, «The Rediscovery of Eastern Jews: German Jews in the East, 1890-1918 », in David Bronsen (éd.), Jews and Germans from 1860 to 1933:The Problematic Symbiosis, Heidelberg, Winter, 1979, p. 338-365; Steven E. Aschheim, Brothers and Strangers: The East European Jew in German and German-Jewish Consciousness, 1800-1923, Madison, The University of Wisconsin Press, 1982; Ulrich Sieg, Jüdische Intellektuelle im Ersten Weltkrieg. Kriegserfahrungen, weltanschauliche Debatten und kulturelle Neuentwürfe, Berlin, Akademie Verlag, 2001.

38. Par exemple, dans le premier volume de la revue: Curt Nawratzki, « Die wirtschaftlichen Lehren des Krieges für Palästina », Der Jude 1 (3), 1916, p. 165-169; ou Davis Trietsch, « Ein Maximalprogramm 
Der Jude représentait l'aboutissement d'un projet de longue date du philosophe Martin Buber (1878-1965), au moins depuis 1903, année où il obtint son doctorat. Après avoir été poussé par Herzl à prendre la direction de la publication de l'hebdomadaire officiel du mouvement, Die Welt, en 1901, il avait d'abord cofondé en 1902 la maison d'édition Jüdischer Verlag. La revue fut d'abord publiée à Vienne par les éditions Richard Löwit de 1916 à 1920, puis par le Jüdischer Verlag à Berlin jusqu'en 1928, et constamment subventionnée par I'Organisation sioniste mondiale, car en elle-même, l'entreprise était déficitaire. Le titre donné au mensuel était repris d'une revue lancée par Gabriel Riesser (18061863), grande figure judéo-allemande du libéralisme et député au parlement de Francfort en 1848, en 1832 à Altona et sous-titrée Ein Journal für GewissensFreiheit. En 1916, la perspective n'était néanmoins plus l'émancipation individuelle du Juif et de sa conscience mais son affirmation nationale comme membre d'un peuple. La revue entendait publier sur ce qui touchait aux intérêts nationaux du judaïsme et véritablement " nationaliser » son lectorat " occidental » assimilé voire assimilationniste, c'est-à-dire, du point de vue sioniste, le « normaliser » tout en le radicalisant ${ }^{39}$.

Il a déjà été montré ailleurs comment Martin Buber et Der Jude ont incorporé et digéré, dans leur propre ligne éditoriale quant à l'identité nationale juive, le nationalisme allemand jusque dans son expression völkisch ${ }^{40}$. On y retrouve le discours sur l'étrangeté fondamentale de l'élément juif (« oriental », « asiatique ») en Allemagne et en Europe ${ }^{41}$. En fait, on y observe surtout le renversement positif de stéréotypes antisémites. La polarité se trouve alors inversée mais l'essentialisme reste présent. Der Jude s'est aussi fait le forum des sempiternels et insolubles questionnements identitaires sur l'essence (Wesen) et les rapports réciproques du Juif et de l'Allemand ${ }^{42}$. Si le but affiché était d'atteindre un équilibre, d'arriver à une synthèse, le postulat d'une différence essentielle entre Juifs et Allemands ne fut, quant à lui, jamais remis en cause.

Du point de vue thématique, Der Jude reposait sur deux piliers : d'une part, les divers aspects et les progrès de l'entreprise sioniste; d'autre part, le dialogue est-ouest au sein du judaïsme. Le nationalisme y était conçu comme

der jüdischen Kolonisation in Palästina », Der Jude 1 (5), 1916, p. 301-308.

39. Voir l'article qui donne le coup d'envoi de la revue : Martin Buber, « Die Losung », Der Jude 1 (1), 1916, p. 1-3. Pour une étude complète de ce périodique, cf. Eleonore Lappin, Der Jude, 1916-1928. Jüdische Moderne zwischen Universalismus und Partikularismus, Tübingen, Mohr, 2000.

40. George L. Mosse, German Jews beyond Judaism, Bloomington, Indiana University Press, 1985; Bernard Susser, « Ideological multivalence. Buber and the German völkisch tradition », Political Theory 5 (1), 1977, p. 75-96; Stefan Vogt, « The First World War, German nationalism, and the transformation of German Zionism », The Leo Baeck Institute Year Book 57 (1), 2012, p. $267-291$; id., Subalterne Positionierungen. Der deutsche Zionismus im Feld des Nationalismus in Deutschland, 1890-1933, Göttingen, Wallstein, 2016.

41. Voir par exemple le célèbre texte de Walther Rathenau (publié sous un pseudonyme-anagramme): W. Hartenau, « Höre, Israel! », Die Zukunft 18, 1897, p. 454-462.

42. Cf. Leo Baeck, Das Wesen des Judentums, Berlin, Nathansen \& Lamm, 1905; Hans Kohn (éd.), Vom Judentum. Ein Sammelbuch, Leipzig, Kurt Wolff, 1913. Voir aussi la controverse opposant Martin Buber à Hermann Cohen en 1916, cf. Lappin, Der Jude, p. 148-167. Sur ce point, voir la contribution de Sonia Goldblum au présent dossier. 
un chemin singulier vers quelque chose de transcendant, dans une perspective cosmopolitique ou messianique qui n'était pas sans rappeler les arguments développés par Johann Gottlieb Fichte (1762-1814) dans ses Discours à la nation allemande ${ }^{43}$. En d'autres termes, dans cette revue s'incarnait le projet ardu de fonder un nationalisme spécifiquement juif à partir des éléments de la tradition juive qui peuvent avoir une portée universelle (le prophétisme, le messianisme, la perpétuelle incitation à la transformation sociale), par exemple dans l'article de Buber intitulé "Zion, der Staat und die Menschheit ${ }^{44}$ », ou celui de Felix Weltsch (1884-1964) intitulé « Die jüdische Renaissance und die Ethik des reinen Willens ${ }^{45}$.» Il en est résulté une tension permanente et irréductible entre particularisme (juif) et universalisme (humain), qui se manifesta notamment après la Première Guerre mondiale dans le discours sur la construction (Aufbau) de la « terre d'Israël » (Eretz Israel) en Palestine, et particulièrement dans la prise en compte de la « question arabe». Pour Buber comme pour d'autres plumes majeures de la revue-Hans Kohn (1891-1971) et Robert Weltsch (1891-1982) par exemple - la coexistence pacifique est alors devenue la pierre de touche de la valeur morale du sionisme et de son entreprise de colonisation, qui devait se distinguer ainsi de celles des grandes puissances coloniales ${ }^{46}$. En un sens, on pourrait dire que les pages ce périodique ont voulu incarner ce « centre spirituel », ce lieu idéal qu'Ahad Haam (1856-1927), grande figure du sionisme en Ukraine, avait appelé de ses vœux ${ }^{47}$. Enfin, Der Jude était aussi le lieu où s'exprimait une certaine tension entre "renaissance juive » et sionisme, puisque le fait qu'il existât des formes de renouveau juif en dehors de ce que proposait le sionisme, c'est-à-dire en Europe, dans la diaspora, pouvait s'avérer problématique dans le cadre d'un discours affirmant que la colonisation juive en Palestine était la seule planche de salut pour les Juifs.

\section{Conclusion}

Les trois exemples développés ici s'articulent autour de trois figures, trois personnalités qu'on peut considérer comme autant d'archétypes allemands : le naturaliste et expert en agronomie; le médecin hygiéniste proposant un diagnostic et une thérapeutique du corps social ou national; le philosophe et éditeur. En jouant sur le titre de l'ouvrage autobiographique de Jakob Wassermann (1873-1934), Mein Weg als Deutscher und Jude ${ }^{48}$ [Mon parcours en tant qu'Allemand et Juif], on pourrait dire qu'il s'est agi en partie de retracer trois parcours en tant que sioniste allemand. En effet, c'est avant tout leur

43. Sur l'importance de Fichte dans le sionisme « culturel », cf. Manfred Voigts, „Wir sollen alle kleine Fichtes werden!" Johann Gottlieb Fichte als Prophet der Kultur-Zionisten, Berlin/ Wien, Philo Verlag, 2003.

44. Der Jude 1 (7), 1916, p. 425-433.

45. Der Jude 5 (4), 1920, p. 220-227.

46. Lappin, Der Jude, p. 435.

47. Sur Ahad Haam et son concept de « centre spirituel », cf. Steven J. Zipperstein, Elusive Prophet: Ahad Ha'am and the Origins of Zionism, Berkeley/ Los Angeles, University of California Press, 1993.

48. Jakob Wassermann, Mein Weg als Deutscher und Jude, Berlin, S. Fischer, 1921. 
cheminement vers l'engagement sioniste qui est intéressant. Dans les trois cas, et peu importe qu'on se focalise sur un homme, une œuvre ou une institution, il convient d'insister sur l'importance cruciale de la socialisation et des formes de sociabilités dans les voies qu'ont empruntées ces parcours. Les milieux d'affaires de Hambourg et les cercles coloniaux dans le cas de Warburg, les associations étudiantes et sportives, les sciences sociales émergentes et le service de santé des armées dans celui de Theilhaber, et enfin les réseaux éditoriaux et académiques dans celui de Buber, ont donné des inflexions déterminantes à ces itinéraires individuels, et permettent de montrer les formes variables qu'a pu prendre, dans le contexte « civilisationnel » allemand, le fait d'être sioniste. 